\title{
1 Efficient abstracting of dive profiles using a broken-stick model
}

2 Short running title: Efficient abstracting of dive profiles

$4 \quad$ Theoni Photopoulou (corresponding author)

5 Centre for Statistics in Ecology, Environment and Conservation, Department of

6 Statistical Sciences, University of Cape Town, Rondebosch 7701, Cape Town, South

7 Africa

8 Email: theoni.photopoulou@uct.ac.za

$9 \quad$ Philip Lovell

10 Sea Mammal Research Unit, Scottish Oceans Institute, University of St Andrews,

11 Scotland KY16 8LB, UK

12 Michael A Fedak

13 Sea Mammal Research Unit, Scottish Oceans Institute, University of St Andrews,

14 Scotland KY16 8LB, UK

15 Len Thomas

16 Centre for Research into Ecological and Environmental Modelling, The Observatory,

17 University of St Andrews, Scotland KY16 9LZ, UK

Jason Matthiopoulos

Institute of Biodiversity, Animal Health and Comparative Medicine, Graham Kerr

20 Building University of Glasgow, Glasgow, Scotland G12 8QQ, UK 


\section{Summary}

27 1. For diving animals, animal-borne sensors are used to collect time-depth

28 information for studying behaviour, ranging patterns and foraging ecology. Often, this

29 information needs to be compressed for purposes of storage or transmission. Widely

30 used devices called Conductivity-Temperature-Depth Satellite Relay Data Loggers

31 (CTD-SRDLs) sample time and depth at high resolution during a dive and then

32 abstract the time-depth trajectory using a broken-stick model (BSM). This

33 approximation method can summarise efficiently the curvilinear shape of a dive,

34 using a piecewise linear shape with a small, fixed number of vertices.

35 2. We present the process of abstracting dives using the BSM and quantify its

36 performance, by measuring the uncertainty associated with the profiles it produces.

37 We develop a method for obtaining a confidence zone and an index for the

38 goodness-of-fit (dive zone index, DZI) for abstracted dive profiles. We validate our

39 results with a case study using dives from elephant seals (Mirounga spp.). We use

40 Generalised Additive Models (GAMs) to determine if the DZI can be used as a proxy

41 for an absolute measure of fit, and investigate the relationship between the DZI and

42 dive shape.

43 3. We found a strong correlation between the residual sum of squares (RSS) for the

44 difference between the detailed and abstracted profiles and the DZI and maximum

45 residual (R4), for dives resulting from CTD-SRDLs (69\% deviance explained). On it's

46 own the DZI explained a lower percentage of deviance which was variable for

47 abstracted dives with different numbers of points. We also found evidence for

48 systematic differences in the DZI for different dive shapes (65\% deviance explained).

49 4. Although the proportional loss of information in the abstraction of time-depth dive

50 profiles by BSM is high, what remains is sufficient to infer goodness-of-fit of the 
51 abstracted profile by reversing the abstraction process. Our results suggest that

52 together the DZI and R4 can be used as a proxy for the RSS, and we present the

53 method for obtaining these metrics for BSM-abstracted profiles.

54

55 Keywords: animal telemetry, broken-stick model, CTD-SRDL, dive profile, dive type,

56 dive zone index, elephant seal, data abstraction

57

58

59

60

61

62

63

64

65

66

67

68

69

70

71

72

73

74

75 


\section{INTRODUCTION}

\section{Data abstraction in animal telemetry: needs and consequences}

78 One of the most effective ways to remotely study movement and behaviour in marine

79 animals is to use animal-borne sensors. Satellite-linked and archival animal

80 telemetry devices have developed rapidly, driven by questions about the behaviour

81 and movement of large vertebrates at sea. A range of purpose-built hardware and

82 software is widely available for deployment on animals. Although animal telemetry

83 devices are able to record information at high temporal and spatial resolution, in

84 many cases devices cannot be recovered, which means the data have to be

85 transmitted. Additionally, it is seldom possible to transmit all data that are collected

86 during a deployment, because the quantity and resolution of the received telemetry

87 data are constrained by several factors: the desired observation time, battery life of

88 the device, bandwidth of the communication system used to relay data, behaviour of

89 the animal, and the software specifications. This means that not all information that

90 is recorded can be recovered. Consequently, data abstraction (defined here as

91 reduction in volume to a simplified representation of the original) is unavoidable for

92 many types of telemetry device.

94 The trade-off between temporal data resolution (i.e., the rate of data sampling and

95 delivery) and the operational longevity of the telemetry device has driven the

96 development of efficient software and memory-saving processing algorithms. One of

97 these is a broken-stick model (BSM), used for the abstraction of two-dimensional

98 dive trajectories (time-depth dive profiles) on-board telemetry devices prior to

99 transmission (Fedak et al. 2002). BSMs are change-point models, falling under

100 piecewise linear approximation, and are used to identify points of abrupt change in 
101 time-series. The piecewise linear profile of a time-series, generated by an efficient

102 linear abstraction method, should have low average deviation from the detailed dive

103 profile. When processing takes place on a small device with limited power for

104 processing and transmissions, it is advantageous to represent piecewise linear

105 profiles using a fixed and small number of bits of information, particularly when using

106 CLS Argos, where message size is fixed (Argos 2011). The algorithm should also be

107 time-efficient, scale linearly in execution time with dive duration, and consistently

108 encode biologically relevant information that might enable inferences on dive

109 function. The BSM fulfils these criteria and was adopted as the default dive

110 abstraction algorithm on CTD-SRDLs in 2006 (pers. comm. Phil Lovell). The

111 predecessor of this model placed breakpoints at locations of maximum inflection in

112 the detailed profile, and while it performed equally well, processing required more

113 time and energy (Fedak, Lovell \& Grant 2001). The BSM was chosen empirically,

114 because it was found to provide a highly satisfactory compromise between the

115 priorities described above. However, its performance has not been formally tested,

116 nor have the consequences of its performance on the biological and ecological

117 conclusions that are drawn in studies using dive profiles abstracted with the BSM.

119 In ecology, change-point models have a long history (MacArthur 1957; MacArthur \&

120 MacArthur 1961) and have seen application in a range of fields, e.g., in

121 oceanography to reduce data volume (Rual 1989), to identify edge effects in plant

122 communities (Toms \& Lesperance 2003), to locate ontogenetic shifts in southern

123 elephant seal diet using stable isotopes (Authier et al. 2012) and in a Bayesian

124 context applied to allometric relationships between tree height and diameter

125 (Beckage et al. 2007). We illustrate the use of the BSM for dive abstraction with a 
126 time-depth dive profile as an example (Figure 1). We call abstracted those dive

127 profiles that have been processed and reduced in resolution using this algorithm. We

128 call detailed those dive profiles that are recorded at regular and frequent time

129 intervals, at the sampling resolution of the device.

130

131 Assessing uncertainty in abstracted dive profiles from elephant seals

132 (Mirounga sp.) as a case study

133 The need for abstraction becomes critical for deployments on wide-ranging marine 134 species, such as seals and turtles, when geographic and temporal data coverage is

135 of interest, and when devices cannot be recovered. For elephant seals and other

136 phocid seals for example, complete time-series of year-round locations and

137 behaviour may be more biologically interesting than detailed information over short

138 periods, and more useful for understanding their life-histories (McConnell, Chambers

139 \& Fedak 1992; Hebblewhite \& Haydon 2010). Until now, the uncertainty associated

140 with abstracted profiles has not been quantified and the implications for ecological

141 studies that use abstracted profiles have not been assessed.

142

143 Abstracted profiles are, by construction, information-poor versions of the detailed

144 trajectories, but since the abstraction process is known in the case of CTD-SRDLs, it

145 is possible to reverse the deterministic steps and retrieve some of the information.

146 Historically, once the abstraction was completed, the high-resolution time-depth

147 profile was overwritten, but current tags store all information that they record, and

148 this can be accessed if the tag is retrieved. Here, we show that it is possible to

149 construct a 100\% confidence zone around an abstracted profile (i.e., upper and

150 lower depth limits at each time point within which the true depth must lie) and 
compare the zone for different dives. This confidence zone is hereafter referred to as

152 the dive zone, and the relative measure of maximum deviation of the abstracted

153 profile, from the detailed profile, is referred to as the dive zone index (DZI).

154

155 The consequences of the abstraction regime by BSM are investigated here using 156 detailed and abstracted dive profiles, from northern ( $M$. angustirostris) and southern

157 elephant seals (M. leonina), as a case study. Elephant seals are large-bodied, long-

158 lived and abundant marine mammals. They spend many months at sea in the open

159 ocean and in coastal or marginal ice zones (McConnell et al. 1992; Jonker \& Bester

160 1998; Campagna et al. 2007; Bailleul et al. 2007; Biuw et al. 2010). They frequently

161 visit high latitudes for extended periods, diving deeply and almost continually,

162 returning to land twice a year to breed and moult. CTD-SRDLs are regularly used in

163 studies of their movement and diving behaviour, and that of other wide-ranging

164 phocid seals.

165

166 The characterisation of dives into types, based on dive parameters, has been a

167 popular approach to the study of diving behaviour (Hindell, Slip \& Burton 1991;

168 Schreer \& Testa 1996; Schreer, Kovacs \& O’Hara Hines 2001; Baechler 2002). In

169 general, the identification of types or groups of behaviour is useful for comparing

170 behavioural patterns and activity budgets between individuals and in different spatial

171 and temporal contexts, and is carried out using a wide range of methods including

172 empirical methods, machine learning, and state-space methods, to name a few (e.g.,

173 Fauchald \& Tveraa 2003; Thums, Bradshaw \& Hindell 2008; McKellar et al. 2014).

174 BSM dives are used widely in studies of diving behaviour and physiology (e.g.,

175 McConnell et al. 1999; Biuw et al. 2007, 2010; Bailleul et al. 2007), without 
176 considering or accounting for uncertainty in the abstracted dive profiles. Ignoring the

177 uncertainty in BSM-derived time-depth profiles may lead to incorrect inferences if the

178 BSM output has substantial error associated with it, and if dives with different shape

179 characteristics differ systematically in the amount of error associated with them. This

180 computationally expedient method has been thought to perform well at capturing

181 biologically relevant aspects of time-depth dive profiles, but this impression has, to

182 date, remained anecdotal.

183

184 Aims and questions

185 In this paper we aim to explain the BSM for dive profile abstraction, provide a

186 method that extracts as much information as possible from abstracted dive data, and

187 improve the interpretability of abstracted dive profiles. To do this we 1) present an

188 overview of the process by which the BSM generates abstracted dive profiles; 2)

189 assess the performance of the BSM for dive profile representation, by comparing

190 detailed and abstracted time-depth dive profiles from elephant seals, as a case

191 study; 3) present a three-step method for obtaining, post hoc, the depth limits on the

192 detailed dive (i.e., the dive zone) based on its BSM abstracted profile; 4) develop an

193 index of goodness-of-fit of abstracted dives (i.e., DZI) and use detailed dive profiles

194 to validate it; and 5) use this index to determine if there are systematic differences in

195 the amount of error associated with different dive types, following (Hindell et al.

196 1991).

197

198 We recast these five aims as research questions: 1) How does the BSM work for

199 dive profile abstraction? How does the representation of the detailed dive change

200 with increasing BSM points? 2) Is the sample of study dives representative? 
201 3) What can we learn from abstracted dives? 4) How is the DZI derived? And, 5) Can 202 the DZI be used as a proxy for the RSS? Does the DZI vary systematically between 203 dive types?

204 205 MATERIALS AND METHODS 206 How does the BSM work for dive profile abstraction?

207 The BSM is an iterative process. For time-depth dive profiles, it is based on 208 minimising the vertical distance (i.e., difference in depth) between the detailed trajectory recorded by the tag and the abstracted dive profile being proposed, at the sampling resolution of the dive (for CTD-SRDLs $4 s, 8 s, 16 s$ or $32 s$ ). We call these vertical distances residuals (Figure 1). The basic principle of the model is that at each iteration, the residuals are calculated, and the point with the biggest residual is added to the abstracted profile. At the first iteration, which we call step zero, the abstracted profile consists only of the start and end points of the dive, forming a straight line at what the tag perceives to be zero depth. This corresponds to a depth buffer at the surface (0-6 m), which is intended to exclude any less interesting shallow undulations from the dive record, that would compete with regular deep dives for transmission. The distance from this straight line to the detailed profile is measured at each time point and the point at which the piecewise linear abstracted profile deviates most from the detailed profile is added to the abstracted profile, creating two new line segments. This is called a breakpoint. This step creates a new piecewise linear profile comprising $I+2$ points, connected by linear segments, and completes one iteration of the model (Figure 1).

225 The maximum residual $\left(R_{I}\right)$ is calculated for each of the resulting line segments and 
226 the point with the greatest departure is selected as the next breakpoint and added to

227 the profile. This process is repeated until the desired number of breakpoints is

228 reached, and the resulting piecewise linear abstracted profile has been constructed.

229 When the abstraction process is complete, the abstracted time-depth dive profile

230 includes $I+2$ time points $\left(T_{1}\right.$ to $\left.T_{I}\right)$, and the corresponding $I$ depth points $\left(D_{1}\right.$ to $\left.D_{I}\right)$.

231 The first and last points, $\left(T_{0}, T_{I+1}, D_{0}\right.$ and $\left.D_{I+1}\right)$ are not transmitted. At $T_{0}$ time is

232 considered to be zero and at $T_{I+1}$ the time elapsed since the beginning of the dive

233 will equal the dive duration. Similarly, at $D_{0}$ and $D_{i+1}$, the depth will both be 0-6 $\mathrm{m}$.

234 The order in which the time-depth points were selected is not stored or transmitted.

235

236 How does the representation of the detailed dive change with increasing BSM 237 points?

238 Using detailed dives from four high-resolution datasets, each representing a 239 continuous dive record from one individual (10943, 12454, 12453 and 12451 in

240 Table 1), we estimated the proportion of high-resolution samples that was

241 represented by the number of BSM points in the corresponding abstracted dive

242 profile (Table 2). We generated an abstracted profile with 3-12 BSM points for each

243 study dive. This resulted in a dataset of 2400 proportions, from 240 dives.

244

245 Is the sample of study dives representative?

246 Four iterations of the BSM are carried out on-board CTD-SRDLs, resulting in a dive

247 profile consisting of six time-depth points: two at the surface, and four at depth, at

248 irregular times, which vary from dive to dive. The number of iterations of the BSM

249 algorithm to be carried out on CTD-SRDLs was chosen as the minimum sufficient

250 number to convey the geometric shape of the dive profile, while keeping the number 
251 of computations low (Fedak et al. 2001).

252

253 We confirmed that the sample of detailed dives from the four individuals was

254 consistent with elephant seal diving behaviour in general. To do this, the DZI was

255 calculated for a sample of 4,000 abstracted dives from 45 southern elephant seals

256 instrumented in four different field seasons (1,000 dives each from two post-moult

257 deployments and two post-breeding deployments; ct40, ct45, ct49, ct58) over two

258 years at the island of South Georgia, South Atlantic (Table 1). The resulting

259 distribution of DZI was visually compared with the distribution of DZI for the detailed

260 dives (Appendix S2, Figures S2.1 and S2.2).

261

262 What can we learn from abstracted dives?

263 The process by which abstracted dive profiles arise when they are collected by CTD-

264 SRDL, is known, therefore it is possible to reverse the deterministic steps and obtain

265 limits to the depth at which the trajectory could have passed, before it was

266 abstracted. The information required to build the dive zone (the $100 \%$ confidence

267 zone for depth) includes the 1) temporal resolution at which the dive was recorded

268 by the tag, the $I+2$ locations, in time and depth (including maximum dive depth), 2)

269 the residual associated with the final, $I+1^{\text {th }}$ breakpoint, and critically, 3 ) the order in

270 which breakpoints were selected during abstraction. The temporal resolution of the

271 detailed dive data is known from the duration of the dive, and the locations are

272 received in the satellite transmissions, but the residuals and the order in which the

273 breakpoints were added need to be determined. Constructing the dive zone involves

274 three steps.

275 
276 First, to find the order in which points were added to the profile, the BSM must be

277 applied to the already abstracted dive trajectory (Figure 2). At each of the $I$ iterations,

278 one of the breakpoints is selected as the point of greatest deviation hence retrieving

279 the order in which the breakpoints were added.

280

281 Second, to determine the limits of the zone, the residuals corresponding to the

282 breakpoints need to be calculated. It is tempting to assume that the residual

283 associated with the $I^{\text {th }}$ breakpoint, $R_{I}$, applies to all segments in the final profile,

284 determining the limits of the dive zone. This is not the case. The limits of the dive

285 zone at each iteration of the model interact with those from previous iterations. As

286 breakpoints are added to the profile, the dive zone changes shape and size.

287 Although the dive zone will always get smaller with subsequent iterations of the

288 algorithm, this geometric effect means that the dive zone needs to be constructed

289 based on all iterations of the model, up to the last one. Furthermore, the resulting

290 dive zone at the final iteration is not symmetric around any segments in a profile and

291 all breakpoints touch the limit of the dive zone (Figure 3).

292

293 The depth points selected by the BSM are coded before transmission according to a

294 pseudo-logarithmic mantissa and exponent representation. With this representation, 295 resolution can be made proportional to the scale of the number being represented, 296 making it useful for depth. Data are then truncated during the decoding process,

297 once they are received. As a consequence, the received depth measurements are

298 binned (i.e. each reported depth has an upper and lower bound) and bin width

299 increases with depth. Bin width is usually smaller than the dive zone height at each

300 breakpoint. As a result, the dive zone is truncated near the deepest point(s) of the 
301 dive where the depths along the line segment near the bottom of the dive exceed the 302 known accuracy of the greatest depth reached during the dive. This is justified since 303 we know that no point in the true profile can be deeper than the maximum depth 304 recorded by the tag. Depth is also truncated at each breakpoint, for the same 305 reason, so the dive zone appears "pinched" at each breakpoint (Figure 3). 306

307 When the $I^{\text {th }}$ iteration is complete, it is known with certainty that there were no points 308 in the detailed trajectory that had a greater residual than the one corresponding to 309 the last point (Figure 2). All other depth points in the true trajectory will now have a 310 smaller vertical distance to the abstracted profile.

312 Third, to construct the dive zone, a number of equally spaced time points need to be 313 selected at which to sample vertical sections of the time-depth space. The resolution 314 of time points should not exceed the resolution at which depth data were collected by 315 the tag. At each of these time points $(t, \ldots, t \operatorname{tmax})$ the estimated lower, $L_{t}$, and upper, $316 U_{t}$, depth bounds define the depth interval through which the true trajectory passed 317 with $100 \%$ confidence (Figure 3). Computer code for all algorithms described was 318 written in $\mathrm{R}$ ( $\mathrm{R}$ Core Team 2013) and can be found in the supplementary material 319 (Appendix S1).

\section{How is the DZI derived?}

322 The approximation of a non-linear path will improve as the number of points $(N)$ that 323 are used to approximate it increases. This should be reflected in a reduction in the 324 size of the maximum residual at the last iteration, as the number of BSM iterations 325 increases. In absolute terms, a small value for $R_{I}$ means that the biggest vertical 
326 outlier in the true path was a small distance away from the abstracted path, which

327 might suggest a better fit. However, $R_{I}$ is affected by the depth, duration and

328 sinuosity of the detailed dive trajectory, and does not follow a strictly decreasing

329 relationship with the number of iterations of the model. When detailed dive data are

330 not available, a reliable and unbiased way of measuring goodness-of-fit is required to

331 assess the accuracy of abstracted profiles, irrespective of depth and duration. On its

332 own $R_{I}$ does not provide an objective way of assessing goodness-of-fit over the

333 whole dive, because it depends on the maximum depth of the dive and the slopes

334 and lengths of the segments that make up the abstracted profile. The mean dive

335 zone height should be a better measure of fit, because it does contain information on

336 the whole dive, and includes the geometric effects that result from the slopes and

337 lengths of the segments that make up the abstracted dive, though importantly, it also

338 does not include information on sinuosity. However, to be comparable between dives

339 it needs to be standardised by dive depth and dive duration. This is the basis for the

340 construction of the DZI.

341

342 The $\mathrm{DZI}$ is calculated using the sum of the differences between the upper, $U$, and

343 lower, $L$, limits of the dive zone at each time step, $t$, in a dive. The sum of these

344 heights is divided by the product of the maximum dive depth, maxdep, and the

345 number of depth points that were recorded by the tag for the dive prior to abstraction,

346 tmax. This quotient ranges between 0 and 1 , where values close to 0 indicate a

347 narrow zone around an abstracted dive and are desirable, and values close to 1

348 indicate a wide zone around an abstracted dive and relatively low confidence in the

349 abstracted dive profile.

$$
D Z I=\frac{\sum_{t=1}^{t \max } U_{t}-L_{t}}{\operatorname{maxdep} * \operatorname{tmax}}
$$


351

352

\section{Can the DZI be used as a proxy for the RSS?}

353

When detailed dive data are available, goodness-of-fit can also be assessed using 354 the sum of squared residuals (RSS) between the detailed and abstracted depths,

$$
R S S=\sum_{t=1}^{t \max }\left(T_{t}-A_{t}\right)^{2}
$$

where $T_{t}$ is the $t^{\text {th }}$ depth in the detailed profile and $A_{t}$ is the $t^{t h}$ depth in the linearly interpolated abstracted profile. The RSS, an absolute measure of fit for detailed dive profiles, was compared to the $\mathrm{DZI}$, the relative measure of fit developed here for abstracted dives, and the biggest residual at the last iteration of the BSM, $R_{I}$ to describe the relationship between them and determine if they could be used as a 361 proxy for the RSS.

To do this, the RSS, DZI and $R_{I}$ were calculated for abstracted profiles with 3-12

364 BSM points for each of the 240 study dives $(n=2400)$. RSS is a non-zero real number, so a Generalized Additive Model (GAM) with a Gamma distribution and a

366 log link function were used, fitted with $\mathrm{mgcv}$ in $\mathrm{R}$ (Wood 2000, 2011). We wanted to

367 know if the DZI could be used as a proxy for the RSS in already abstracted dives

368 received by CTD-SRDL, but we were also interested to know if the amount of 369 deviance explained by the DZI changed with increasing breakpoints. Hence, we

370 fitted a model to a subset of the data representing six breakpoints, and did model

371 selection to find the best model, but also fitted a model to a subset of the data 372 representing each number of breakpoints explored in this study (three to twelve) 
373 including only DZI as a covariate without doing model selection.

374

375 In the first case, RSS was modelled with DZI and $R_{4}$ as explanatory variables, and 376 individual seal as a random effect. In the second case, RSS was modelled with DZI 377 as the only explanatory variable, and individual seal as a random effect, as above. In 378 both cases, the relationship between the RSS and the covariates was non-linear, so 379 they were fitted as smooth functions with a shrinkage smoother ("cs") as the basis 380 function and k=4 knots. The number of knots was found to be sufficient using 381 standard mgcv checks. This basis allows for the smooth coefficients to be shrunk to 382 zero and effectively removed from the model when there is no relationship with the 383 response. We specified a gamma parameter value of 1.4 to reduce the chance of 384 overfitting. The random effect was fitted using the "re" smooth, as described above. 385 We used restricted maximum likelihood (REML) as the fitting method (Wood 2011).

\section{Does the DZI vary systematically between dive types?}

388 The availability of detailed dive data (depth sampled every 4 s) made it possible to 389 investigate the effects of the abstraction process, develop methods to reverse the 390 abstraction and quantify goodness-of-fit. Detailed data were taken from a high

391 temporal resolution dataset recorded by a specially configured archival SRDL 392 deployed on a northern elephant seal after the moult at Año Nuevo, California, and 393 recovered six months later (Table 1). These dives were chosen by eye, as being 394 representative of the six functional or behavioural characterisations sometimes used

395 to classify elephant seal dives into types; U-shaped dives (U), V-shaped dives (V), 396 square-bottom dives (SQ), wiggle dives (W), root-shaped dives (R) and drift dives 397 (DR), after Hindell, Slip, and Burton (1991). Ten dives of each type from each 
398 individual were included in the case study. No detailed dive data from southern 399 elephant seals were used, but several datasets of abstracted dives were available. A 400 sample of over 22,000 abstracted dives that had already been classified into types 401 were used to investigate systematic differences in goodness-of-fit between dive 402 types (Table 1). 403

404 The classification of abstracted dives into types was done using the random forest 405 tree-building method (Breiman 2001). Random forest is a machine learning tool; we 406 used an implementation in the randomForest library in R (Liaw \& Wiener 2002). A 407 supervised version of the method was used to classify dives, whereby 3,000 dives, $40814 \%$ of the dataset, were classified based on visual cues, and used to train the 409 remaining dives. The overall "out-of-bag" error, an unbiased estimate of classification 410 error, was $3.6 \%$. This represents the aggregate of the prediction error rate at each 411 bootstrap iteration (Liaw \& Wiener 2002). The variables supplied to the function for 412 classification were maximum dive depth, bathymetry and fifteen dive parameters 413 (Photopoulos 2007). This method has been found to work well for dive classification, 414 using both detailed and abstracted dive data (Thums et al. 2008).

416 The $\mathrm{DZI}$ and $R_{4}$ were also calculated for the each dive in the dataset. The $\mathrm{DZI}$ used 417 as the response variable in GAM with a quasibinomial distribution and a logit link 418 function, dive type as a factor variable, $R_{4}$ as a smooth covariate using "cs" basis 419 function ( $k=4$ knots, checked as above) and individual animal as a simple random 420 effect using the "re" smooth. The model was fitted using the REML method and 421 gamma was specified as 1.4 , as above. 
423 Model assessment was done based on inspection of the residuals, the relationship

424 between the observations and the fitted values for each model, and the percentage 425 of deviance explained.

426

\section{RESULTS}

428 In the introduction, we outlined five questions relating to the BSM for dive profile 429 abstraction, the usefulness of the DZI as a goodness-of-fit measure and the validity 430 of our assessment of it as such: 1) How does the BSM work for dive profile 431 abstraction? How does the representation of the detailed dive change with 432 increasing BSM points? 2) Is the sample of study dives representative? 3) What can 433 we learn from abstracted dives? 4) How is the DZI derived? And, 5) Can the DZI be 434 used as a proxy for the RSS? Does the DZI vary systematically between dive types?

436 1) The BSM algorithm for dive profile abstraction is illustrated in Figure 1 and 437 animated in Appendix S2 of the supplementary material. The proportion of detailed 438 samples in a dive increases with the number of breakpoints in the abstracted profile, 439 and depends on dive duration. Overall, the proportion of a dive represented by its 440 abstracted profile is very low, starting at $1 \%$ with three breakpoints and reaching $4 \%$ 441 with twelve, and increases by a constant $0.33 \%$ for each breakpoints added (Table

442 2). The mean relationship was similar for all animals but there were differences in the 443 variability in the relationship (Figure 4).

445 2) Variability between the samples of abstracted and detailed dives was expected, 446 due to individual variability and the different regions where data were collected. 447 However, visual comparison of the distributions of DZI from the different samples did 
448 not suggest any striking differences (Appendix S2, Figures S2.1 and S2.2,

449 Supplementary material).

450

4513 ) Even though the detailed trajectory cannot be recovered unless the device is

452 physically recovered, the order in which breakpoints were added to the profile and

453 the $100 \%$ confidence limits to the detailed profile, which we call the dive zone, can

454 be calculated from abstracted dives. This makes it possible to derive the DZI (Figure $4553)$.

456

457 4) The derivation of the DZI is based on the maximum depth of the dive, its duration 458 and the upper and lower limits to the dives zone.

459

460 5) There was a strong, positive relationship between the RSS and the DZI together 461 with $R_{4}$. We found that the $\mathrm{DZI}, R_{4}$ and a random effect for individual explained $69 \%$ 462 of the variability in the RSS for abstracted profiles with six breakpoints (deviance 463 explained) (Figure 5). On its own, the DZI explained a variable proportion of 464 deviance for abstracted dives with differing numbers of breakpoints, but there was an 465 overall positive relationship with increasing breakpoints for dives with four or more 466 breakpoints (Figure 6). The DZI varied substantially between dive type and had an 467 increasing relationship with $R_{4}$ (Figure 7). The dive type associated with the biggest 468 DZI values were square dives (SQ) and both V-shaped (V) and drift dives (DR) had 469 the smallest DZI, under the model (Figure 8). Dive type and $R_{4}$ together explained $47065 \%$ of the variability in the DZI (deviance explained), having accounted for 471 individual variability by fitting a random effect for individual. 


\section{DISCUSSION}

474 Change-points models are used in many fields, from neuroscience and epidemiology

475 to genetics and finance, to identify changes in time-series. One of these, the BSM,

476 was adopted on board CTD-SRDLs, as a working solution to the problem of linearly

477 approximating a non-linear path in the vertical dimension with as little information as

478 possible, while aiming to retain biologically relevant content. Until now, the

479 approximation error associated with abstracted profiles had not been investigated.

480 We provide a way of calculating and summarizing the error associated with dive

481 profiles derived using the BSM, in order to assess the information content of

482 abstracted dives. Our results suggest that BSM-abstracted dive profiles do, in fact,

483 retain enough information to estimate goodness-of-fit of the abstracted profile to the

484 detailed profile. The strong, positive relationship between the DZI and $R_{4}$, and the

485 RSS is evidence for this. It means that researchers using BSM-abstracted dive

486 profiles to make inference about animal behaviour and diving ecology can now

487 calculate the $\mathrm{DZI}$ and $R_{4}$ and incorporate a relative measure of error into their

488 analyses.

489

490 With the BSM, as with other linear approximation methods, the number of iterations

491 is critical to the quality of the abstracted dive. Our results confirm the findings of a

492 preliminary investigation during tag development, regarding the adequacy of different

493 numbers of iterations, that resulted in the standard use of four iterations of the

494 algorithm for dives collected by CTD-SRDLs. That study found that the information

495 gained in the transmission of a fifth breakpoint is relatively small, and that it would be

496 more useful to receive a measure of the variance of the detailed dive, either for the

497 whole dive, or each segment, which is supported by the results presented in this 
498 paper.

499

500 It is worth noting that although the BSM can efficiently summarise a curvilinear 501 trajectory, using a piecewise-linear shape, profiles resulting from low iteration 502 numbers, may mask important biological features even if they closely approximate 503 the detailed trajectory. Our methods do not provide means for assessing the 504 biological content of abstracted dives, since the detail lost, however small, might be 505 the most biologically interesting. For example, a useful feature of the BSM is that it is 506 efficient at identifying long sections where the trajectory has low variability. In the 507 case of dives, these are often the descent and ascent phases, leaving only two 508 points to confer information about the bottom phase of the dive, which is arguably 509 the most interesting biologically. Dives with low variability in change in depth in the 510 bottom phase, also have the lowest DZI, as we found here for drift dives (DR type).

511 However, when classifying abstracted dives with a method like a random forest 512 algorithm, ancillary behavioural data are necessary for validating classes as being 513 functionally distinct, in addition to being phenomenologically distinct.

514

515 Large numbers of dive profiles are collected using CTD-SRDLs from many different 516 species (over 21 million profiles since 1991, SMRU 2012, unpublished data) and 517 used to make inferences about the biology and behaviour of the instrumented 518 animals. It seems essential that a method for assessing the accuracy of these 519 abstracted dives, at least statistically if not biologically, is made widely available. The 520 methods we have presented here make that possible. They also provide a way of 521 carrying out a "pilot" analysis when detailed dive data are available. The differences 522 in diving behaviour between species, and habitats, may render different numbers of 
523 breakpoints appropriate for the questions being asked. When detailed dive data are

524 available, the result of dive abstraction with different number of iterations can be

525 investigated to achieve the best result for a specific study, prior to deployment.

526 Together, these uses for our methods may help make more robust the behavioural

527 conclusions we can draw from telemetry data.

528

529 More generally, through this work we have developed a method for quantifying

530 uncertainty in the fitted values for BSMs, when the original time-series is no longer

531 available. This method could be applied to any situation where the original time-

532 series data are not longer available. This could be useful in situations where large

533 amounts of data are being generated and cannot be stored or transmitted at the

534 original resolution. We demonstrate that measures of fit like the DZI and $R_{4}$ have a

535 strong correspondence to the RSS and could therefore be used instead.

536

537 ACKNOWLEDGEMENTS

538 This work was supported by SMRU Ltd (now SMRU Marine) in the form of a PhD

539 fellowship (T.P.). Completion of the manuscript was supported by a National

540 Research Foundation Scarce Skills Postdoctoral Fellowship at the University of

541 Cape Town, South Africa (T.P.). The CTD-SRDL data presented in this manuscript

542 were collected as part of a project funded by the Natural Environment Research

543 Council (NERC) grants NE/E018289/1 and NER/D/S/2002/00426. The authors are

544 extremely grateful for access to three high-resolution datasets from Kerguelen

545 Islands that were made available by Christophe Guinet and collected under the SO-

546 MEMO framework. Fieldwork was carried out according to the Animals (Scientific

547 Procedures) Act 1986 guidelines and approved by the University of St Andrews 
548 Animal Welfare Committee. The authors thank Martin Biuw (Akvaplan-niva, Trømso,

549 Norway) and Lars Boehme (SMRU) for use of the CTD-SRDL data, Nora Hanson

550 (SMRU) and Lars Boehme for useful comments on the manuscript, Samantha

551 Gordine (SMRU) for testing the code, and two anonymous reviewers for their

552 constructive comments on the manuscript.

553

554 DATA ACCESSIBILITY

555 - R scripts: uploaded as supporting material

556 - Example data of detailed and abstracted dive data: uploaded as supporting

$557 \quad$ material

558 - For access to the full datasets used in this study please contact the data

559 owners directly. South Georgia Island and Año Nuevo deployments: Mike

$560 \quad$ Fedak (maf3@st-andrews.ac.uk) and Lars Boehme (lb284@st-

561 andrews.ac.uk). Kerguelen Islands deployments: Christophe Guinet

562 (Christophe.GUINET@cebc.cnrs.fr).

563

564 REFERENCES

565 Aoki, K., Watanabe, Y., Crocker, D., Robinson, P.W., Biuw, M., Costa, D., Miyazaki, 566 N., Fedak, M.A. \& Miller, P.J.O. (2011) Northern elephant seals adjust gliding 567 and stroking patterns with changes in buoyancy: validation of at-sea metrics of 568 body density. The Journal of experimental biology, 214, 2973-87.

569 Argos. (2011) Argos User's Manual. URL http://wwwargos-systemorg/files/pmedia/p 570 ublic/r363_9_argos_manual_en.pdf 
571 Authier, M., Martin, C., Ponchon, A., Steelandt, S., Bentaleb, I. \& Guinet, C. (2012)

$572 \quad$ Breaking the sticks: a hierarchical change-point model for estimating

573 ontogenetic shifts with stable isotope data. Methods in Ecology and Evolution, 3,

$574 \quad 281-290$.

575 Baechler, J. (2002) Dive shapes reveal temporal changes in the foraging behaviour

576 of different age and sex classes of harbour seals (Phoca vitulina). Canadian

577 Journal of Zoology, 80, 1569-1577.

578 Bailleul, F., Charrassin, J.-B., Monestiez, P., Roquet, F., Biuw, M. \& Guinet, C.

579 (2007) Successful foraging zones of southern elephant seals from the

$580 \quad$ Kerguelen Islands in relation to oceanographic conditions. Philosophical

581 transactions of the Royal Society of London. Series B, Biological sciences, 362,

$582 \quad 2169-81$.

583 Beckage, B., Joseph, L., Belisle, P., Wolfson, D.B. \& Platt, W.J. (2007) Bayesian

584 change-point analyses in ecology. The New Phytologist, 174, 456-67.

585 Biuw, M., Boehme, L., Guinet, C., Hindell, M., Costa, D., Charrassin, J.-B., Roquet,

586 F., Bailleul, F., Meredith, M., Thorpe, S.E., Tremblay, Y., McDonald, B., Park,

587 Y.-H., Rintoul, S.R., Bindoff, N., Goebel, M., Crocker, D., Lovell, P., Nicholson,

588 J., Monks, F. \& Fedak, M.A. (2007) Variations in behavior and condition of a

589 Southern Ocean top predator in relation to in situ oceanographic conditions.

$590 \quad$ Proceedings of the National Academy of Sciences of the United States of

$591 \quad$ America, 104, 13705-10. 
592 Biuw, M., Nøst, O., Stien, A., Zhou, Q., Lydersen, C. \& Kovacs, K. (2010) Effects of 593 hydrographic variability on the spatial, seasonal and diel diving patterns of 594 southern elephant seals in the eastern Weddell Sea. PloS one, 5, 1-14.

Breiman, L. (2001) Random Forests. Machine Learning, 45, 5-32.

596

597

598

599

600

601

602

603

604

605

606

607

608

609

610

611

Campagna, C., Piola, A.R., Marin, M.R., Lewis, M., Zajaczkovski, U. \& Fernández, T. (2007) Deep divers in shallow seas: Southern elephant seals on the Patagonian shelf. Deep Sea Research Part I: Oceanographic Research Papers, 54, 17921814.

Fauchald, P. \& Tveraa, T. (2003) Using first-passage time in the analysis of arearestricted search and habitat selection. Ecology, 84, 282-288.

Fedak, M.A., Lovell, P. \& Grant, S. (2001) Two approaches to compressing and interpreting time-depth information as collected by time-depth recorders and satellite-linked data recorders. Marine Mammal Science, 17, 94-110.

Fedak, M.A., Lovell, P., McConnell, B. \& Hunter, C. (2002) Overcoming the constraints of long range radio telemetry from animals: getting more useful data from smaller packages. Integrative and comparative biology, 42, 3-10.

Hebblewhite, M. \& Haydon, D.T. (2010) Distinguishing technology from biology: a critical review of the use of GPS telemetry data in ecology. Philosophical transactions of the Royal Society of London. Series B, Biological sciences, 365 , 
612 Hindell, M., Slip, D. \& Burton, H. (1991) The diving behaviour of adult male and 613 female southern elephant seals, Mirounga leonina (Pinnipedia: Phocidae). 614 Australian Journal of Zoology, 39, 595-619.

615 Jonker, F. \& Bester, M. (1998) Seasonal movements and foraging areas of adult 616 southern female elephant seals, Mirounga leonina, from Marion Island. Antarctic 617 Science, 10, 21-30.

618 Liaw, A. \& Wiener, M. (2002) Classification and regression by randomForest. $R$ $619 \quad$ News, 2, 18-22.

620 MacArthur, R. (1957) On the relative abundance of bird species. Proceedings of the 621 National Academy of Sciences of the United States of America, 43, 293-295.

622 MacArthur, R. \& MacArthur, J. (1961) On bird species diversity. Ecology, 42, 594$623 \quad 598$.

624 McConnell, B., Chambers, C. \& Fedak, M.A. (1992) Foraging ecology of southern 625 elephant seals in relation to the bathymetry and productivity of the Southern 626 Ocean. Antarctic Science, 4, 393-398.

627 McConnell, B., Fedak, M.A., Lovell, P. \& Hammond, P. (1999) Movements and 628 foraging areas of grey seals in the North Sea. Journal of Applied Ecology, 36, $629 \quad 573-590$.

630 McKellar, a. E., Langrock, R., Walters, J.R. \& Kesler, D.C. (2014) Using mixed 631 hidden Markov models to examine behavioral states in a cooperatively breeding 632 bird. Behavioral Ecology, 00, 1-10. 
633 Photopoulos, T. (2007) Behavioural Changes of a Long-Ranging Diver in Response 634 to Oceanographic Conditions. University of St Andrews.

635 R Core Team. (2013) R: A language and environment for statistical computing. R $636 \quad$ Foundation for Statistical Computing.

637 Rual, P. (1989) For a better XBT bathy-message on board quality control, plus a new 638 data reduction method. Western Pacific International Meeting and Workshop on 639 Toga Coare (eds J. Picaut, R. Lukas \& T. Delcroix), pp. 823-833. Noumea, New $640 \quad$ Caledonia.

641 Schreer, J., Kovacs, K. \& O'Hara Hines, R. (2001) Comparative diving patterns of 642 pinnipeds and seabirds. Ecological Monographs, 71, 137-162.

643 Schreer, J. \& Testa, J. (1996) Classification of Weddell seal diving behavior. Marine $644 \quad$ Mammal Science, 12, 227-250.

645 Thums, M., Bradshaw, C.J.A. \& Hindell, M.A. (2008) A validated approach for 646 supervised dive classification in diving vertebrates. Journal of Experimental 647 Marine Biology and Ecology, 363, 75-83.

648 Toms, J. \& Lesperance, M. (2003) Piecewise regression: a tool for identifying 649 ecological thresholds. Ecology, 84, 2034-2041.

650 Wood, S.N. (2000) Modelling and smoothing parameter estimation with multiple 651 quadratic penalties. Journal of the Royal Statistical Society: Series B (Statistical $652 \quad$ Methodology), 62, 413-428. 
653 Wood, S.N. (2011) Fast stable restricted maximum likelihood and marginal likelihood

654 estimation of semiparametric generalized linear models. Journal of the Royal

655 Statistical Society: Series B (Statistical Methodology), 73, 3-36.

656 
Figures and Tables

Table 1. Deployment information and morphometrics for the detailed datasets from a northern and three southern elephant seal (a sample of 60 study dives was taken from the dive record of each seal), and the abstracted dataset(s) from 45 southern elephant seals. For "ct" deployments, length is given as the mean (standard error) of all animals of each sex. For deployments 12454 , 12453, 12451, two length measurements were available for each animal, so we present the mean (standard error) of the two measurements. Only one length measurement was made during deployment 10943.

\begin{tabular}{|c|c|c|c|c|c|c|c|}
\hline Deployment & Species & Deployment location & Period (UTC) & Sampling regime & Morphometrics & Sex & Dive duration \\
\hline 10943 & $\begin{array}{l}\text { M. angustirostris } \\
1 \text { adult male }\end{array}$ & Año Nuevo CA, USA & $\begin{array}{l}23 / 08 / 2008 \text { to } \\
16 / 02 / 2009\end{array}$ & $\begin{array}{l}\text { Time and depth } \\
\text { every } 4 \mathrm{~s} *\end{array}$ & $\begin{array}{l}\text { Length: } 350.0 \mathrm{~cm} \\
\text { Axial Girth: } 295.0 \mathrm{~cm}\end{array}$ & M & $24.95 \min (0.77)$ \\
\hline 12454 & $\begin{array}{l}\text { M.leonina } \\
1 \text { adult female }\end{array}$ & Kerguelen Islands & $\begin{array}{l}30 / 10 / 2012 \text { to } \\
15 / 02 / 2013\end{array}$ & $\begin{array}{l}\text { Time and depth } \\
\text { every } 4 \mathrm{~s}\end{array}$ & $\begin{array}{l}\text { Length: } 235.5 .0 \mathrm{~cm}(6.5) \\
\text { Weight: } 258 \mathrm{~kg}\end{array}$ & $\mathrm{~F}$ & $19.18 \min (0.63)$ \\
\hline 12453 & $\begin{array}{l}\text { M.leonina } \\
1 \text { adult female }\end{array}$ & Kerguelen Islands & $\begin{array}{l}20 / 10 / 2012 \text { to } \\
11 / 02 / 2013\end{array}$ & $\begin{array}{l}\text { Time and depth } \\
\text { every } 4 \mathrm{~s}\end{array}$ & $\begin{array}{l}\text { Length: } 271.5 \mathrm{~cm}(6.5) \\
\text { Weight: } 425 \mathrm{~kg}\end{array}$ & $\mathrm{~F}$ & 23.48 cm (0.67) \\
\hline 12451 & $\begin{array}{l}\text { M.leonina } \\
1 \text { adult female }\end{array}$ & Kerguelen Islands & $\begin{array}{l}30 / 10 / 2012 \text { to } \\
15 / 02 / 2013\end{array}$ & $\begin{array}{l}\text { Time and depth } \\
\text { every } 4 \mathrm{~s}\end{array}$ & $\begin{array}{l}\text { Length: } 234.0 \mathrm{~cm}(1.0) \\
\text { Weight: } 275 \mathrm{~kg}\end{array}$ & $\mathrm{~F}$ & $18.52 \min (0.64)$ \\
\hline ct1 & $\begin{array}{l}\text { M. leonina } \\
6 \text { adult females }\end{array}$ & $\begin{array}{l}\text { Husvik, } \\
\text { South Georgia Island }\end{array}$ & $\begin{array}{l}06 / 01 / 2004 \text { to } \\
25 / 08 / 2004\end{array}$ & CTD_GEN_07B* & Length: $242.5 \mathrm{~cm}(6.7)$ & $\mathrm{F}$ & $23.96 \min (0.07)$ \\
\hline ct8 & $\begin{array}{l}\text { M. leonina } \\
7 \text { adult females } \\
4 \text { adult males }\end{array}$ & $\begin{array}{l}\text { Husvik, } \\
\text { South Georgia Island }\end{array}$ & $\begin{array}{l}13 / 01 / 2005 \text { to } \\
31 / 10 / 2005\end{array}$ & CTD_GEN_07B & $\begin{array}{l}\text { Length: } 255.7 \mathrm{~cm}(2.0) \\
\text { Length: } 301.3 \mathrm{~cm}(8.5)\end{array}$ & $\begin{array}{l}\mathrm{F} \\
\mathrm{M}\end{array}$ & $36.59 \min (0.25)$ \\
\hline ct40 & $\begin{array}{l}\text { M. leonina } \\
5 \text { adult females } \\
5 \text { adult males }\end{array}$ & $\begin{array}{l}\text { Husvik, } \\
\text { South Georgia Island }\end{array}$ & $\begin{array}{l}28 / 01 / 2008 \text { to } \\
09 / 12 / 2008\end{array}$ & CTD_GEN_07B & $\begin{array}{l}\text { Length: } 250.5 \mathrm{~cm}(13.0) \\
\text { Length: } 343.6 \mathrm{~cm}(24.4)\end{array}$ & $\begin{array}{l}\mathrm{F} \\
\mathrm{M}\end{array}$ & $27.47 \min (0.38)$ \\
\hline ct45 & $\begin{array}{l}\text { M. leonina } \\
10 \text { adult females }\end{array}$ & $\begin{array}{l}\text { Husvik, } \\
\text { South Georgia Island }\end{array}$ & $\begin{array}{l}17 / 10 / 2008 \text { to } \\
01 / 02 / 2009\end{array}$ & CTD_GEN_07B & Length: $248.7 \mathrm{~cm}(2.9)$ & $\mathrm{F}$ & $19.77 \min (0.18)$ \\
\hline ct49 & $\begin{array}{l}\text { M. leonina } \\
11 \text { adult females } \\
1 \text { adult male }\end{array}$ & $\begin{array}{l}\text { Husvik, } \\
\text { South Georgia Island }\end{array}$ & $\begin{array}{l}28 / 01 / 2009 \text { to } \\
12 / 01 / 2010\end{array}$ & CTD_GEN_07B & $\begin{array}{l}\text { Length: } 240.5 \mathrm{~cm}(4.0) \\
\text { Length: } 237.00 \mathrm{~cm}\end{array}$ & $\begin{array}{l}\mathrm{F} \\
\mathrm{M}\end{array}$ & $25.92 \min (0.35)$ \\
\hline
\end{tabular}




\begin{tabular}{llllllll}
\multirow{2}{*}{ ct58 } & M. leonina & Husvik, & 22/10/2009 to & CTD_GEN_07B & Length: $249.5 \mathrm{~cm}(3.2)$ & $\mathrm{F}$ & $18.97 \mathrm{~min}(0.19)$ \\
& 13 adult females & South Georgia Island & $05 / 02 / 2010$
\end{tabular}

*CTD_GEN_07B is the parameter specification with which the instruments were programmed.

** This tag sampled time and depth at $1 \mathrm{~Hz}$ but resolution was reduced to one sample every $4 \mathrm{~s}$ for consistency other timedepth data. The tag operated on a three-day duty cycle ( 3 days on, 3 days off). 
Table 2. Observed mean proportion of detailed depth samples from individuals $10943,12454,12453,12451$ represented by an abstracted profile of a given number of BSM points (standard error, SE), and mean DZI (standard error, SE). The grey row highlights the case of a dive profile with 6 points, produced by 4 iterations of the BSM.

\begin{tabular}{ccc}
\hline $\begin{array}{c}\text { Number of BSM } \\
\text { points }\end{array}$ & $\begin{array}{c}\text { Mean proportion of depth } \\
\text { samples represented by } \\
\text { abstracted profile (SE) }\end{array}$ & Mean DZI (SE) \\
\hline 3 & $0.010(0.0002)$ & $0.993(0.0002)$ \\
4 & $0.013(0.0003)$ & $0.549(0.0156)$ \\
5 & $0.017(0.0003)$ & $0.303(0.0103)$ \\
6 & $0.020(0.0004)$ & $0.158(0.0048)$ \\
7 & $0.023(0.0005)$ & $0.110(0.0036)$ \\
8 & $0.027(0.0005)$ & $0.085(0.0030)$ \\
9 & $0.030(0.0006)$ & $0.071(0.0026)$ \\
10 & $0.033(0.0007)$ & $0.060(0.0023)$ \\
11 & $0.037(0.0007)$ & $0.051(0.0019)$ \\
12 & $0.040(0.0008)$ & $0.044(0.0017)$ \\
\hline
\end{tabular}



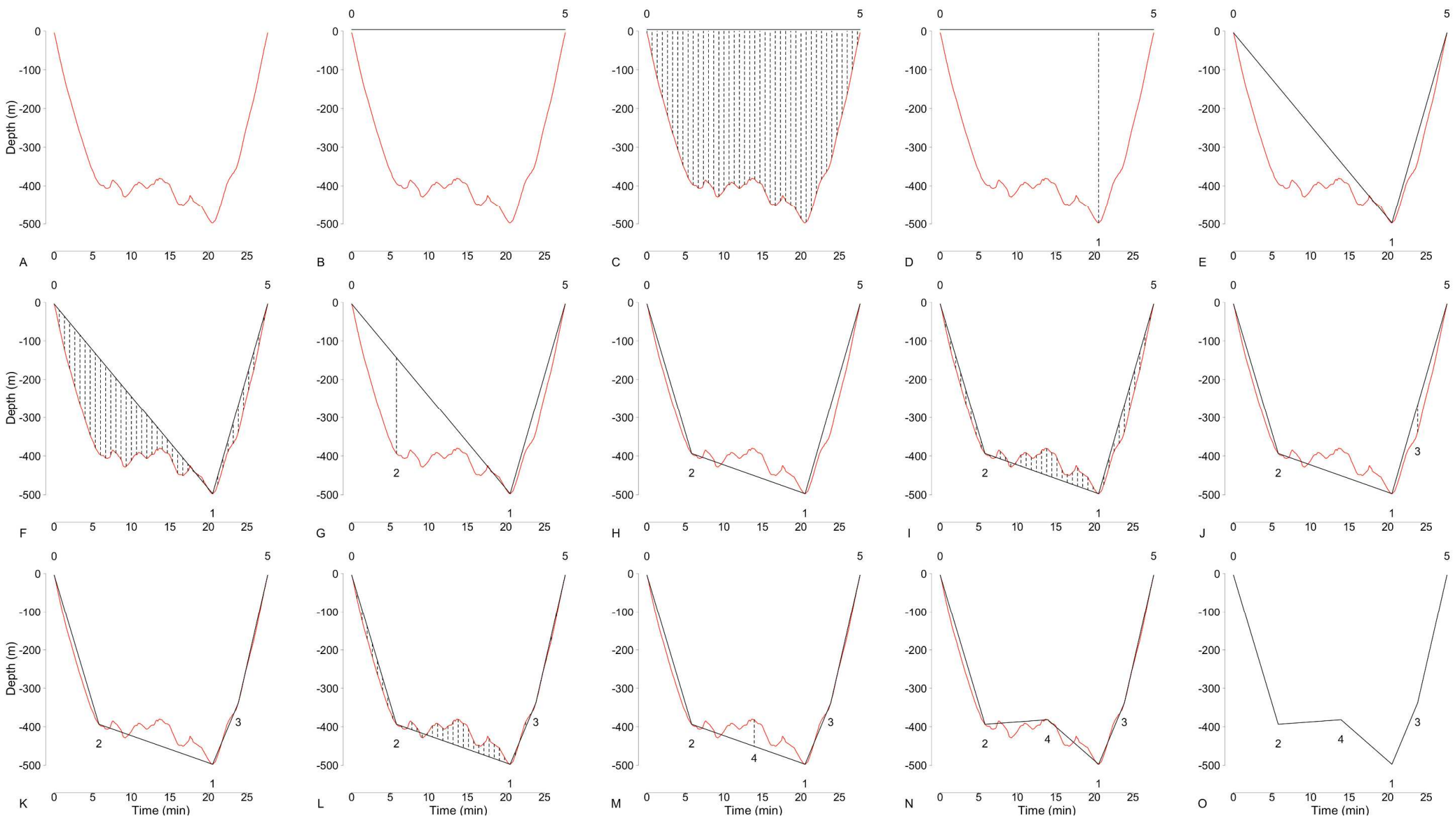

Figure 1. Step-wise illustration of time-depth dive profile abstraction for a dive from elephant seal 10943 (see Table 1) using a broken-stick model. With this abstraction regime, highly resolved time-depth measurements are processed to generate abstracted dive profiles made up of $I+1$ consecutive line segments that approximate the true, non-linear time-depth trajectory travelled. This is achieved through $I(=4$, here) iterations of the algorithm. The red lines represent the true time-depth dive path, the solid black lines represent the abstracted dive path and the dashed lines represent the residuals for the abstracted dive 
path, at each iteration of the algorithm. The numbers represent the order in which points are added to the abstracted profile. Points 0 and 5 mark the beginning and end of the dive. The dashed black lines represent the residuals that are calculated at each time step between the true and abstracted dive. 

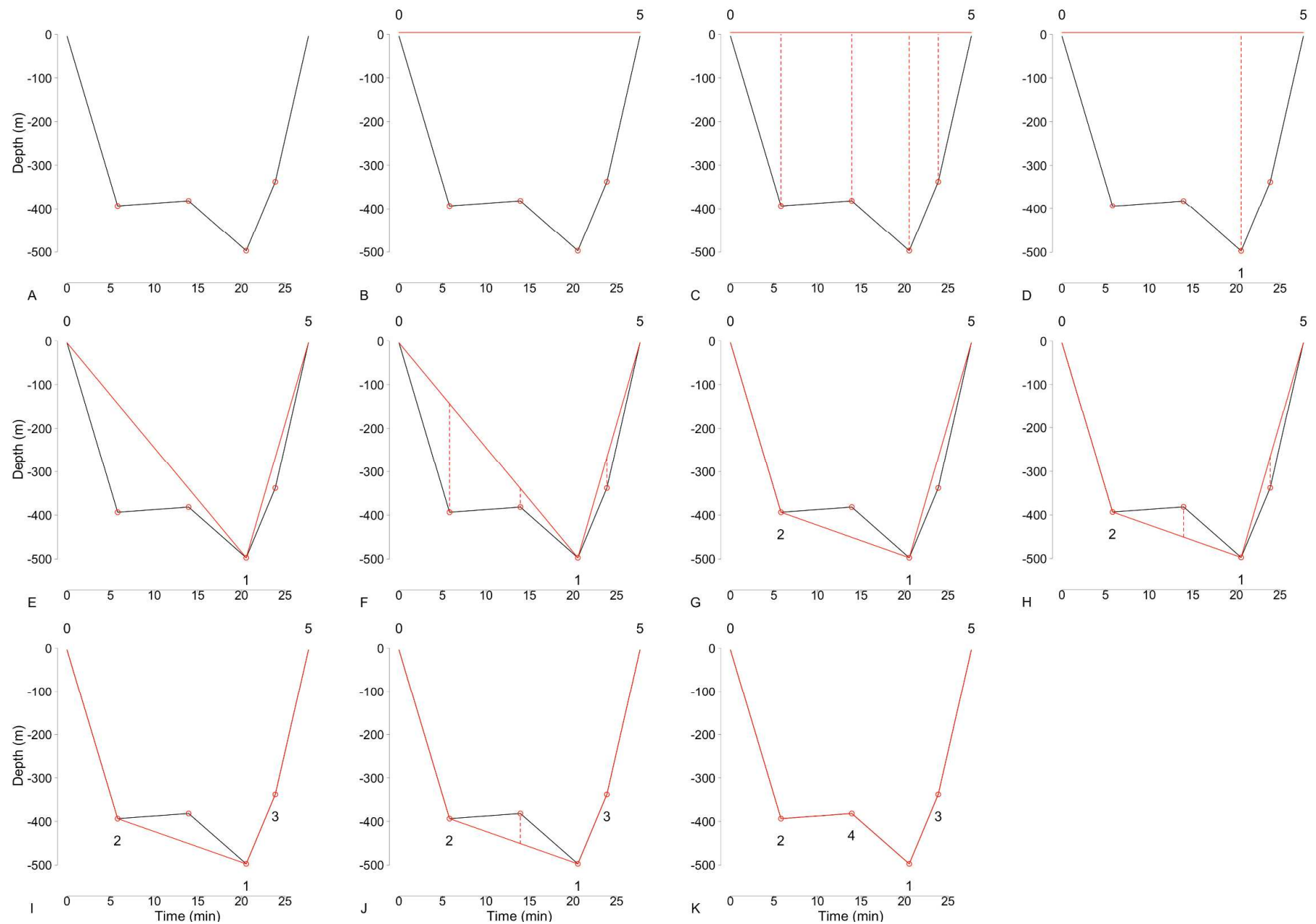

Figure 2. Step-wise illustration of the calculation of the order in which breakpoints were added to a time-depth dive profile abstracted using the BSM, received from CTD-SRDLs telemetry devices. The black line represents the abstracted time-depth dive profile received from the device. The red points represent known breakpoints. The solid red lines the proposed BSM abstracted dive path at each iteration of the model, and the dashed red lines represent the residuals that correspond to known breakpoints. 

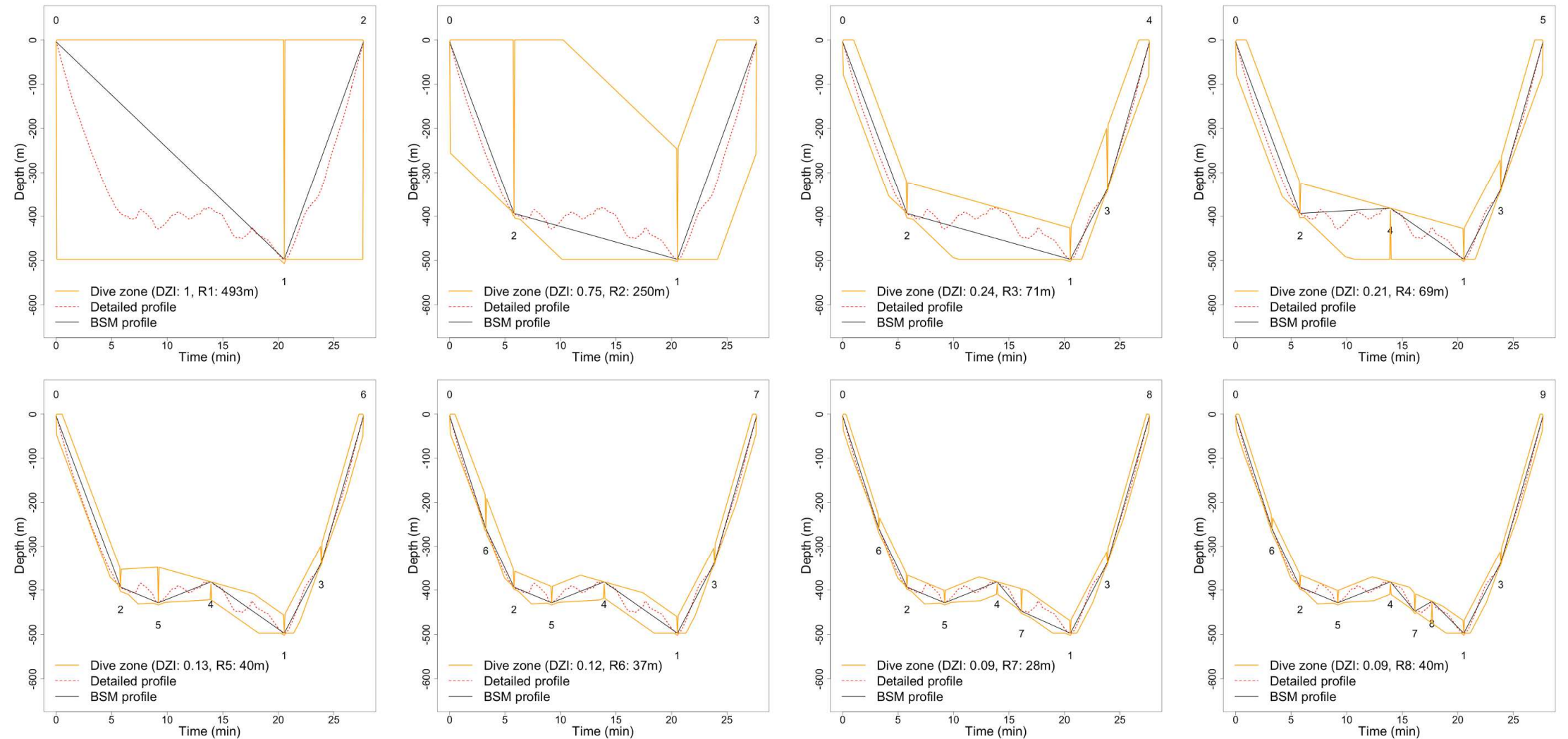

Figure 3. Step-wise illustration of the construction of the dive zone and its evolution as points are added to the abstracted dive profile. We use an example dive from individual 10943 (see Table 1), with up to ten iterations of the broken-stick model, i.e., using 3-12 points, in total. 


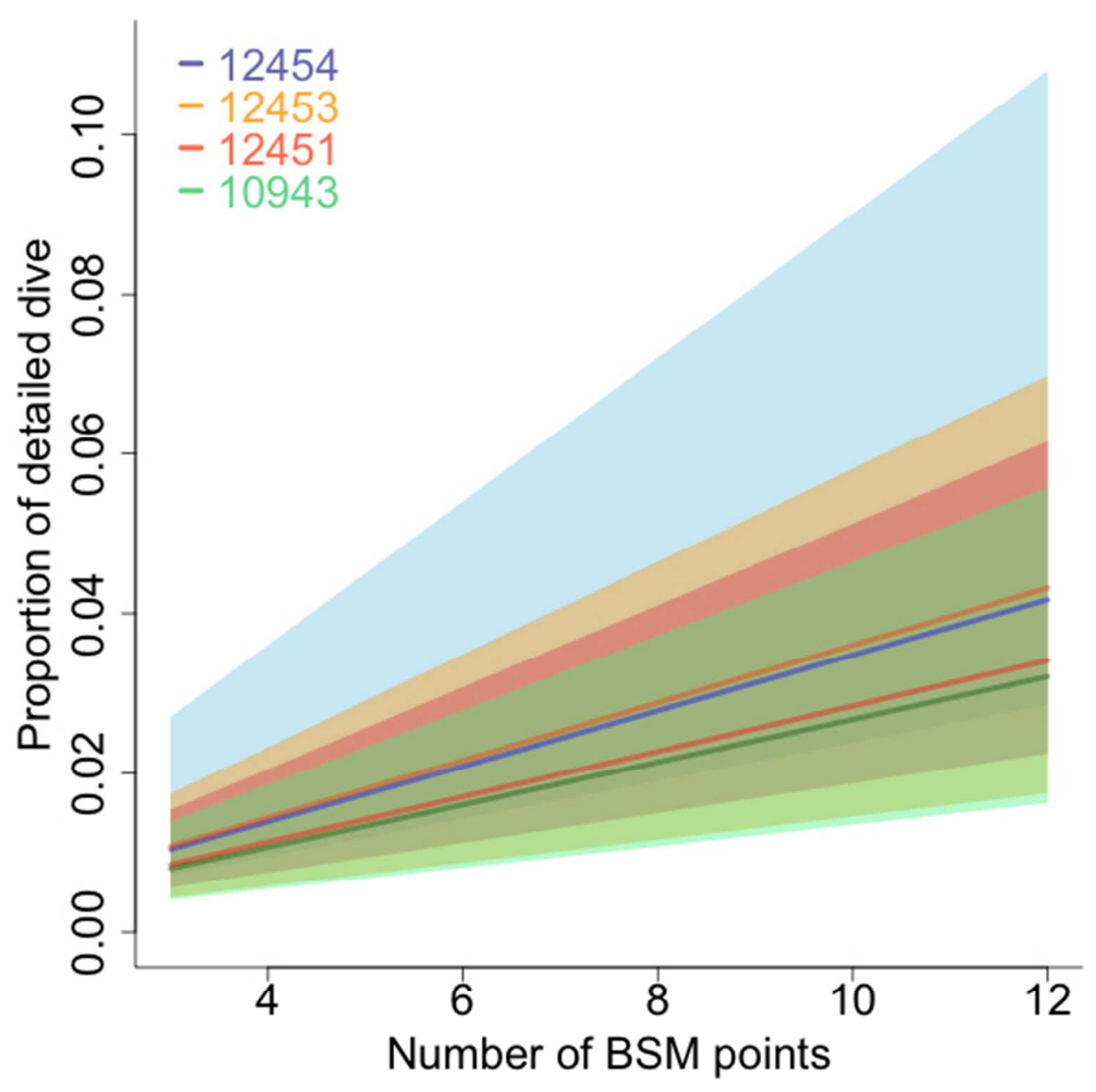

Figure 4. The relationship between the proportion of high-resolution time-depth samples and the number of BSM points in the abstracted dive profiles of 240 case study dives from a northern elephant seal and three southern elephant seals (see Table 1). Abstracted profiles with 3-12 points in total were generated for each of the study dives and compared with the full resolution profile. The coloured areas incudes minimum and maximum observed range of the relationship for each individual. $211 \times 211 \mathrm{~mm}(72 \times 72 \mathrm{DPI})$ 

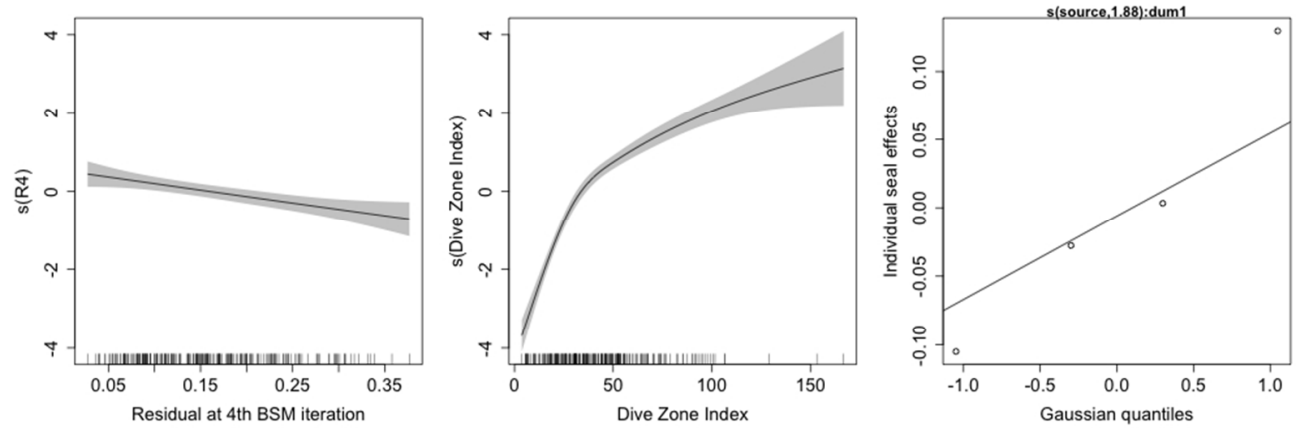

Figure 5. Smooth functions of the covariates in a Generalized Additive Model with log(RSS) as the response, DZI a smooth covariate, and individual dive as a random effect. The data used to fit this model were abstracted dive profiles of 4,000 study dives from 45 southern elephant seals instrumented at South Georgia Island in 2008 and 2009. The grey area includes two standard errors for the fitted relationship. $352 \times 117 \mathrm{~mm}(72 \times 72 \mathrm{DPI})$ 


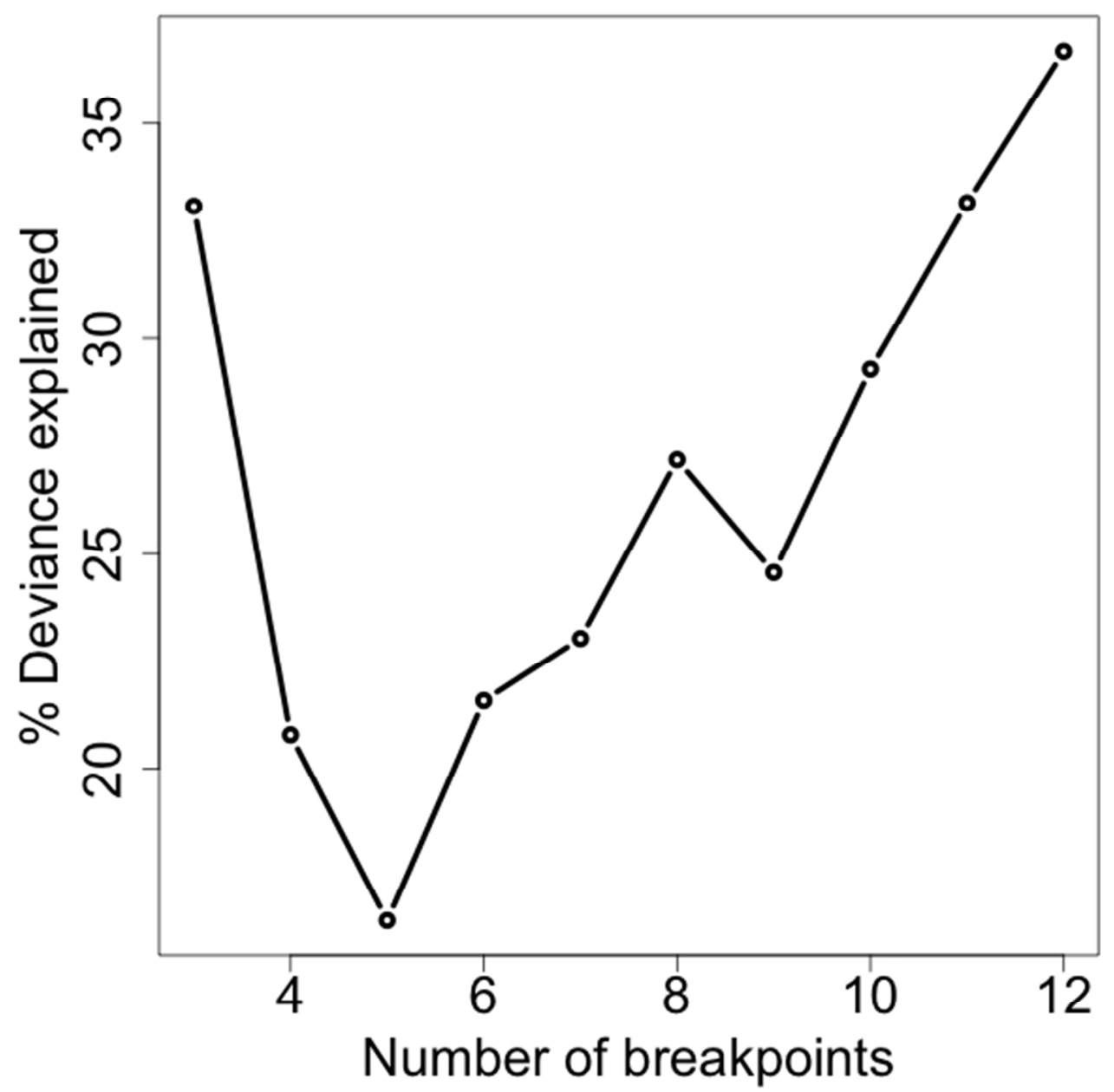

Figure 6. The relationship between the percentage of deviance explained by a model for RSS with DZI as the explanatory variable and individual seal as a random effect, and the number of breakpoints in the dives being considered.

$211 \times 211 \mathrm{~mm}(72 \times 72 \mathrm{DPI})$ 

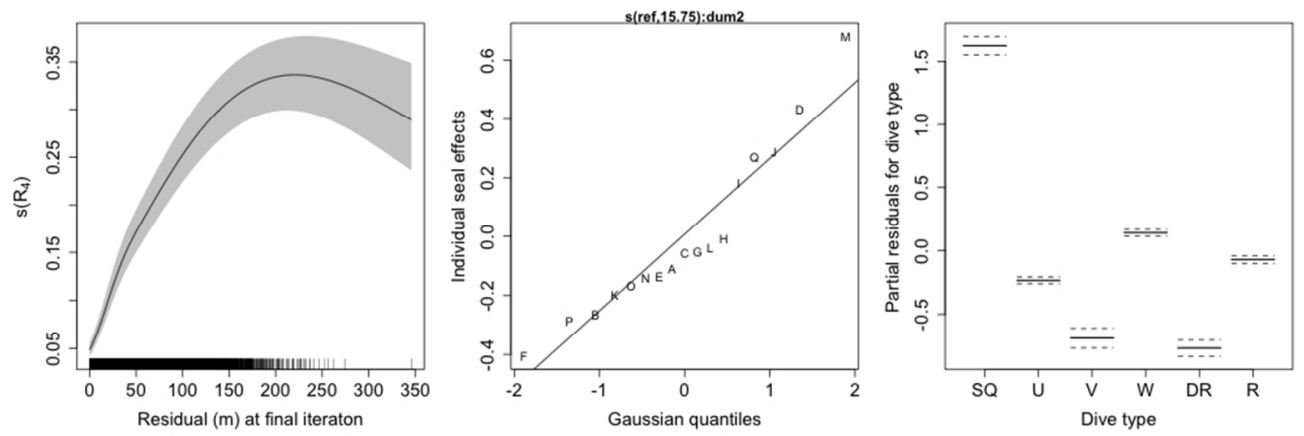

Figure 7. Smooth functions of the covariates in a Generalized Additive Model with dive zone index as the response, dive type as a factor variable, the residual as the final iteration of the BSM as a smooth covariate, and individual seal as a random effect. The data used to fit this model were abstracted dive profiles of 22,305 study dives from 17 southern elephant seals instrumented at South Georgia Island in 2004 and 2005. The grey area includes two standard errors for the fitted relationship.

$352 \times 117 \mathrm{~mm}(72 \times 72 \mathrm{DPI})$ 


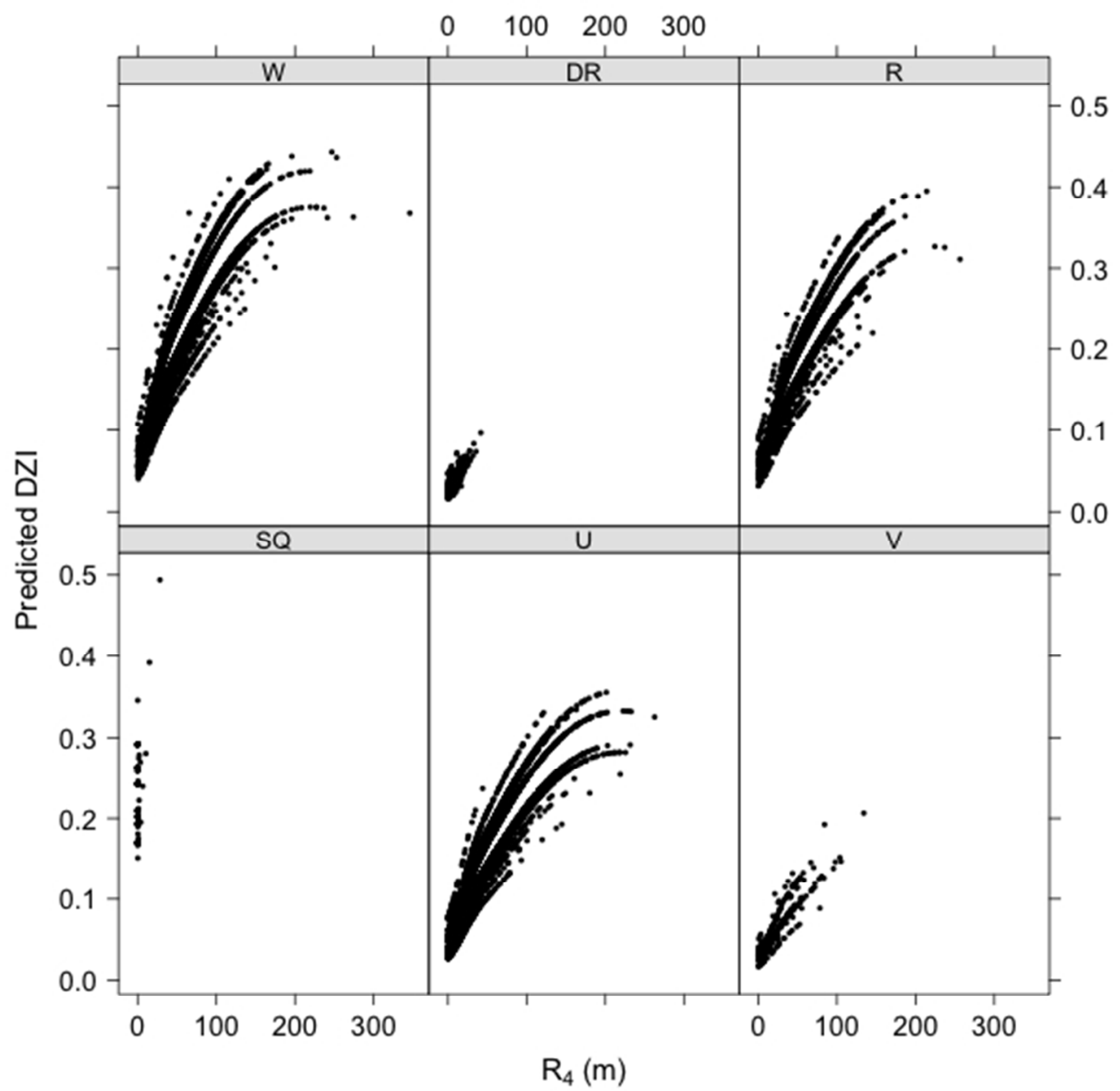

Figure 8. The fitted relationship between the dive zone index (DZI) and the residual at the final interaction of the broken stick model (R4) for each dive type. These are the predictions based on a Generalized Additive Model, with R4 as smooth covariate, dive type as a factor variable, and individual seal as a random effect.

The data used to fit this model were abstracted dive profiles of 22,305 study dives from 17 southern elephant seals instrumented at South Georgia Island in 2004 and 2005. $211 \times 211 \mathrm{~mm}(72 \times 72 \mathrm{DPI})$ 